\title{
Carcinoma de paratireoide: um relato de caso
}

\author{
Parathyroid carcinoma: case report
}

Matheus Henrique de Freitas Silva ${ }^{1}$, Pedro Otávio de Resende Morais ${ }^{1}$, Thaís Andrade de Souza ${ }^{1}$, André Luis Ribeiro Muniz², Bárbara C Silva², Thiago Bechara Noviello², Marcella Menezes Andrade ${ }^{3}$

\begin{abstract}
RESUMO
O carcinoma de paratireoide (CP) constitui entidade patológica incomum e detentora de peculiaridades propedêutico-terapêuticas. Em geral, apresenta-se como hiperparatireoidismo primário sintomático, no qual corresponde a somente $1 \%$ nesse subgrupo. O diagnóstico de CP é difícil, tanto clínico quanto histológico, devido à inconstância de critérios. Laboratorialmente, é comum apresentar hipercalcemia e PTH bastante acima do valor de referência. A cirurgia é o único tratamento curativo e efetivo para o carcinoma de paratireoide. Relato de caso: Paciente M.P.R.L., 48 anos, sexo feminino, melanodérmica, natural e procedente de Felisburgo - MG. Evoluíra há 02 anos com fraqueza progressiva, anemia, disfunção renal, dores crônicas e perda ponderal (cerca de $15 \mathrm{~kg}$ nos últimos cinco meses), associada a hipercalcemia PTH-dependente e declínio da função renal. Estudo ultrassonográfico (USG) de região cervical demonstrou presença de dois nódulos tireoideanos e cintilografia de paratireoides sem evidências de hiperplasia ou adenoma das glândulas. Foi admitida no Hospital Santa Casa de Belo Horizonte para seguimento propedêutico e terapêutico do quadro apresentado.
\end{abstract}

Palavras-chave: Hormônio Paratireóideo. Hipercalcemia. Hiperparatireoidismo Primário.

\begin{abstract}
Parathyroid carcinoma (PC) is an uncommon pathology which has propaedeutic and therapeutic peculiarities. In general, it presents as symptomatic primary hyperparathyroidism, that corresponds to only $1 \%$ in this subgroup. Both the clinical and histological diagnosis of PC are difficult due to inconsistent criteria. Laboratorial exams commonly present hypercalcemia and PTH well-above the reference value. Surgery is the only curative and effective treatment for parathyroid carcinoma. Case report: Patient M.P.R.L., 48 years old, female, melanodermic, native and from Felisburgo - MG. The disease had been progressing for 2 years with progressive weakness, anemia, renal dysfunction, chronic pain and weight loss (about $15 \mathrm{~kg}$ in last five months), associated with PTH-dependent hypercalcemia and renal function decline. A cervical study showed the presence of two thyroid nodules whereas a parathyroid scintigraphy showed no evidence of glands hyperplasia or adenoma. She was admitted to the Santa Casa Hospital of Belo Horizonte for a propaedeutic and therapeutic management of the presented profile.
\end{abstract}

Key-words: Parathyroid Hormone. Hypercalcemia. Hyperparathyroidism, Primary.

1. Graduando de Medicina, Universidade de Itaúna (UIT)

2. Médico preceptor do serviço de Endocrinologia da Santa Casa de Belo Horizonte - SCBH

3. Residente do serviço de Endocrinologia da Santa Casa de Belo Horizonte - SCBH
CORRESPONDÊNCIA:

Matheus Henrique de Freitas Silva Santa Casa de Belo Horizonte Av Francisco Sales, 1111 CEP 30150-221, Belo Horizonte-MG

Recebido em 18/07/2017 Aprovado em 27/02/2018 


\section{Introdução}

O carcinoma de paratireoide (CP) constitui neoplasia rara e corresponde a menos de $1 \%$ dos casos de hiperparatireoidismo primário. ${ }^{1} \mathrm{~A}$ história natural é de uma neoplasia com crescimento lento e progressivo, porém com alto índice de recidiva. Acomete indivíduos na quinta década de vida, sem predileção por sexo. ${ }^{2}$

Idade avançada ao diagnóstico, sexo masculino e metástases à distância são indicadores prognósticos deficientes. O tamanho do tumor e o status linfonodal não são preditivos de sobrevida global. ${ }^{3}$ O comportamento biológico do CP é muitas vezes indolente e diversos estudos de dados retrospectivos mostraram taxas de sobrevivência em 10 anos variando de 50 a $70 \% .4$

O diagnóstico de CP é difícil, tanto clínico quanto histológico, devido à inconstância de critérios. ${ }^{5}$ Os sintomas clínicos decorrem do aumento do paratormônio (PTH) sendo, em sua maioria, constitucionais, ósseos e renais. Na presença de glândula paratireoide palpável e PTH aumentado, devese suspeitar de carcinoma. Laboratorialmente, é comum apresentar hipercalcemia e PTH bastante acima do valor de referência (VR). ${ }^{6}$

Hodiernamente, a cirurgia é o único tratamento curativo e efetivo para o carcinoma de paratireoide. Remoção do tumor "em bloc" e de linfonodos aumentados, bem como a lobectomia ipsilateral da tireoide constituem os objetivos do ato cirúrgico. ${ }^{7}$

\section{Relato de caso}

Paciente de 48 anos, sexo feminino, melanodérmica, natural e procedente de Felisburgo - MG e hipertensa há 10 anos. Evoluíra há dois anos com fraqueza progressiva, anemia, disfunção renal, dores crônicas e perda ponderal (cerca de $15 \mathrm{~kg}$ nos últimos cinco meses). Ao exame físico não apresentava nódulo ou massa cervical palpável. Apresentava exames laboratoriais demonstrando hipercalcemia PTH-dependente cujos valores eram Cálcio Iônico (CaI): 2,61 mmol/L (VR: 1.20-1.40 mmol/ L), PTH: $1.137 \mathrm{pg} / \mathrm{mL}$ (VR: $10-65 \mathrm{pg} / \mathrm{mL})(25 / 07 /$ 16); Cálcio Total (CaT): 17,2mg/dL (VR: 8,8-10,6 $\mathrm{mg} / \mathrm{dL})(13 / 09 / 16) ;$ Fosfatase Alcalina: 722U/L (VR: 30-120 U/L) (16/09/16). A disfunção renal já havia sido evidenciada previamente a hospitalização, em que a creatinina elevou-se de $0,8 \mathrm{mg} / \mathrm{dL}$ em 04/ 2014 para 2,0 mg/dL em 05/2015. Estudo ultrassonográfico (USG) de região cervical (em 12/05/16) demonstrou presença de dois nódulos tireoideanos, um em lobo direito de $3,3 \times 2,6 \mathrm{~mm}$ e outro em lobo esquerdo de $7,0 \times 4,3 \mathrm{~mm}$. Cintilografia de paratireoides (em 30/08/16) com ${ }^{99 m} \mathrm{mc}-M D P$ não demonstrou hiperplasia ou adenoma das glândulas. USG abdominal (em 09/09/16) evidenciou colelitíase e micronefrolitíase, que não havia sido demonstrada em exame de 09/05/16.

Foi admitida, então, em 15/10/2016 no Hospital Santa Casa de Belo Horizonte para investigação de hipercalcemia PTH-dependente. Foram solicitados exames laboratoriais na admissão cujos resultados foram: PTH: $1.754 \mathrm{pg} / \mathrm{mL}$ (VR: $10-65 \mathrm{pg} /$ $\mathrm{mL}$ ); CaI: $2,49 \mathrm{mmol} / \mathrm{L}$ (VR: 1,20-1,40 mmol/L); CaT: $15,7 \mathrm{mg} / \mathrm{dL}$ (VR: $8,8-10,6 \mathrm{mg} / \mathrm{dl}$ ) ; Creatinina: $2,49 \mathrm{mg} / \mathrm{dL}$ (VR: $0,6-1,2 \mathrm{mg} / \mathrm{dL}$ ); Albumina: $3,3 \mathrm{~g} / \mathrm{dL}$ (VR: 3,5-5,2 g/dL). Definido como hiperparatireoidismo primário, indicou-se a paratireoidectomia que foi realizada em 24/10/16. No ato cirúrgico foi notado aumento volumétrico da glândula superior esquerda, de cerca de $2 \mathrm{~cm}$ (o dobro do volume em relação às demais), de aspecto nodular e calcificado. Um fragmento da paratireoide superior direita foi retirado e implantado no subcutâneo no antebraço direito. A dosagem do PTH intraoperatório não foi realizada.

O resultado do anatomopatológico da paratireoide superior esquerda evidenciou nódulo capsulado contendo áreas de células com núcleos aumentados e focos de necrose; observando-se ainda, alterações degenerativas e invasão vascular (Figuras 1 e 2). A microscopia das demais glândulas não evidenciou anormalidades. Diante dos achados, não se pôde precisar a distinção entre adenoma e carcinoma, concluindo-se como histologia compatível com neoplasia de paratireoide.

Após ato cirúrgico, paciente apresentou acidose metabólica com ânion gap normal e manutenção dos níveis séricos de PTH $(1.587 \mathrm{pg} / \mathrm{mL}$ - VR: 10-65 pg/mL), CaT (11,7 mg/dL - VR: 8,8 a 10,6 $\mathrm{mg} / \mathrm{dL})$ e CaI $(2,11 \mathrm{mmol} / \mathrm{L}$ - VR: $1,20-1,40 \mathrm{mmol} /$ L) ainda no Centro de Terapia Intensiva. Porém, evoluiu com boa recuperação e mantida internada para investigação. 


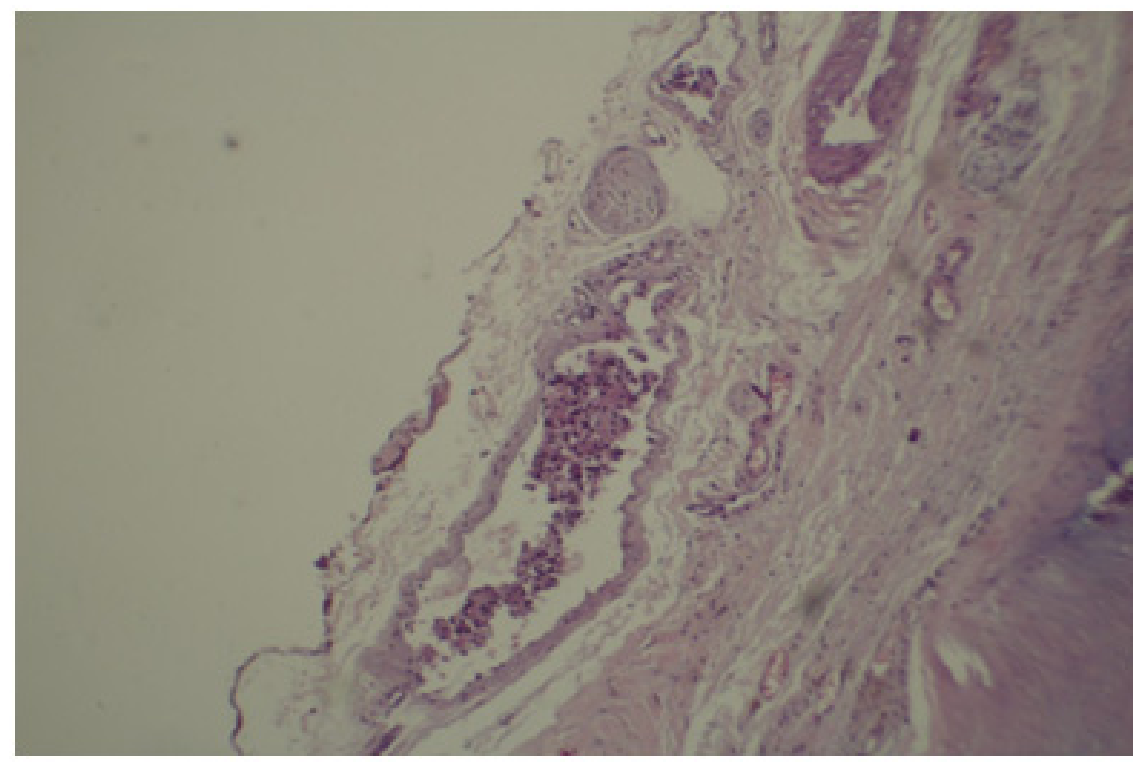

Figura 1: Lâmina histológica demonstrando invasão vascular pela lesão.

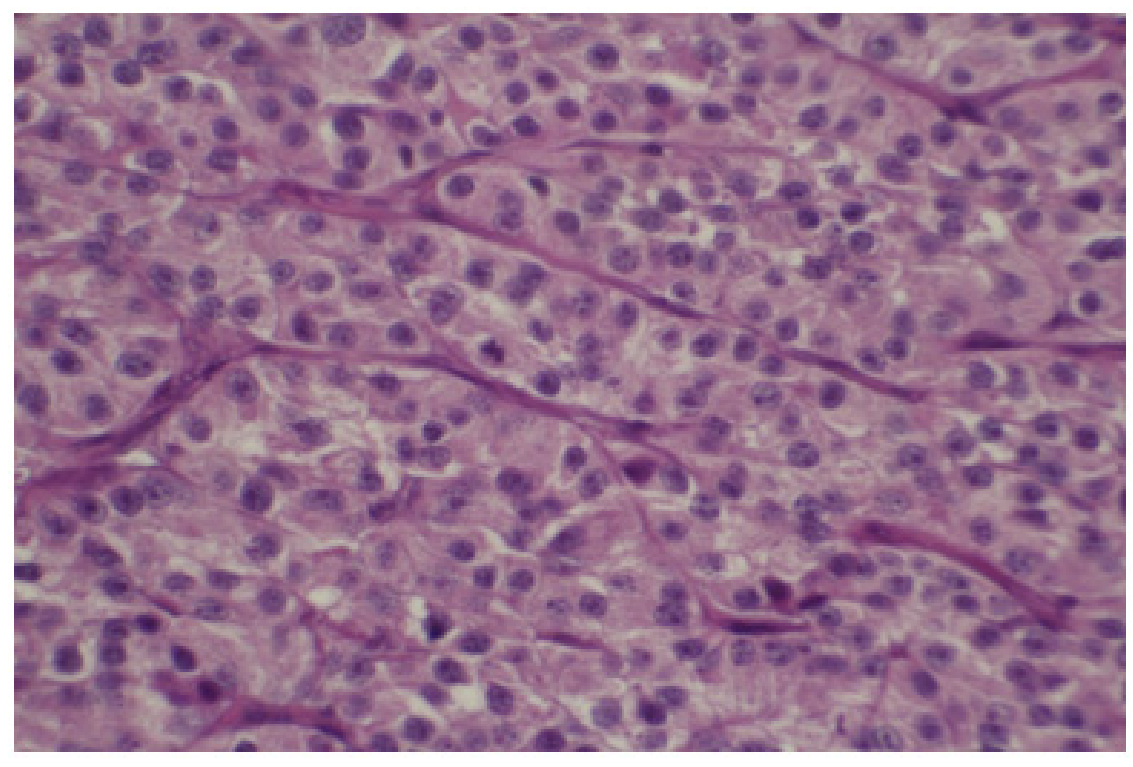

Figura 2: Lâmina histológica da paratireoide demonstrando presença de figuras de mitose.

Realizou-se nova cintilografia de paratireoides com ${ }^{99 m T c-M D P ~ e m ~ 03 / 11 / 16 ~ q u e ~ n a ̃ o ~ e v i d e n c i o u ~}$ adenoma/hiperplasia dessas glândulas, porém presença de hipercaptações em maxilar inferior, úmeros proximais e fêmures proximais. Estudo tomográfico de crânio em 07/11/16 evidenciou lesão medular do osso zigomático direito questionando-se a possibilidade de um tumor marrom (osteoclastoma), rarefação óssea da calota craniana e calcificações puntiformes nos nervos ópticos bilaterais. Tomografia de abdome e pelve, da mesma data, demons- trou rarefação óssea difusa associada a lesões osteolíticas em ossos pélvicos e lesão osteolítica heterogênea em $3^{\circ}$ arco costal esquerdo de aspecto distinto das demais observadas, que poderia corresponder a variante do tumor marrom ou lesão de etiologia neoplásica distinta.

Para progressão propedêutica realizou-se (em 10/11/16) biópsia guiada por tomografia computadorizada da lesão osteolítica em arco costal. Do material obtido dosou-se o nível de PTH, cujo valor foi de $15.314 \mathrm{pg} / \mathrm{mL}$. 
Desse modo, foi proposta exérese da lesão em $3^{\circ}$ arco costal, realizada em 21/11/16, por toracostomia com drenagem pleural fechada e reconstrução com retalho de músculo peitoral e serrátil. A análise anatomopatológica da lesão demonstrou presença de adenocarcinoma metastático caracterizado pela presença de figuras de mitose, hipercromasia com núcleos pleomórficos e invasão de tecido fibroadiposo (Figuras 3, 4 e 5).

A paciente no pós-operatório imediato apre- sentou PTH (em 23/11/16) de 237 pg/mL (VR: 10$60 \mathrm{pg} / \mathrm{mL}$ ) e cursou com quadro de hipocalcemia. Necessitou reposição de cálcio associado a calcitriol, sugerindo a ocorrência de "fome óssea". Intercorreu, também, com acidose metabólica hiperclorêmica, elevando progressivamente as escórias renais, sem oligúria ou choque. Permaneceu por 16 dias no CTI, onde foi tratado o distúrbio hidroeletrolítico, com estabilização do estado geral e manutenção de proteinúria subnefrótica e glicosúria.

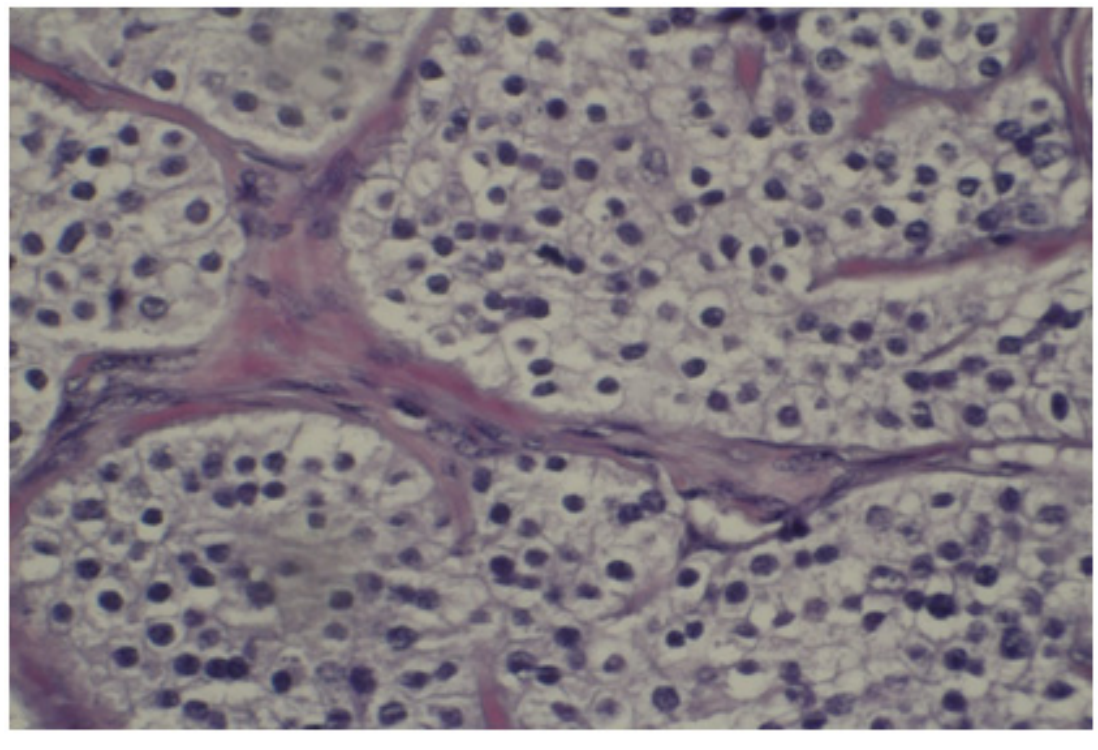

Figura 3 : Lâmina histológica demonstrando presença de figuras de mitose na lesão de arco costal.

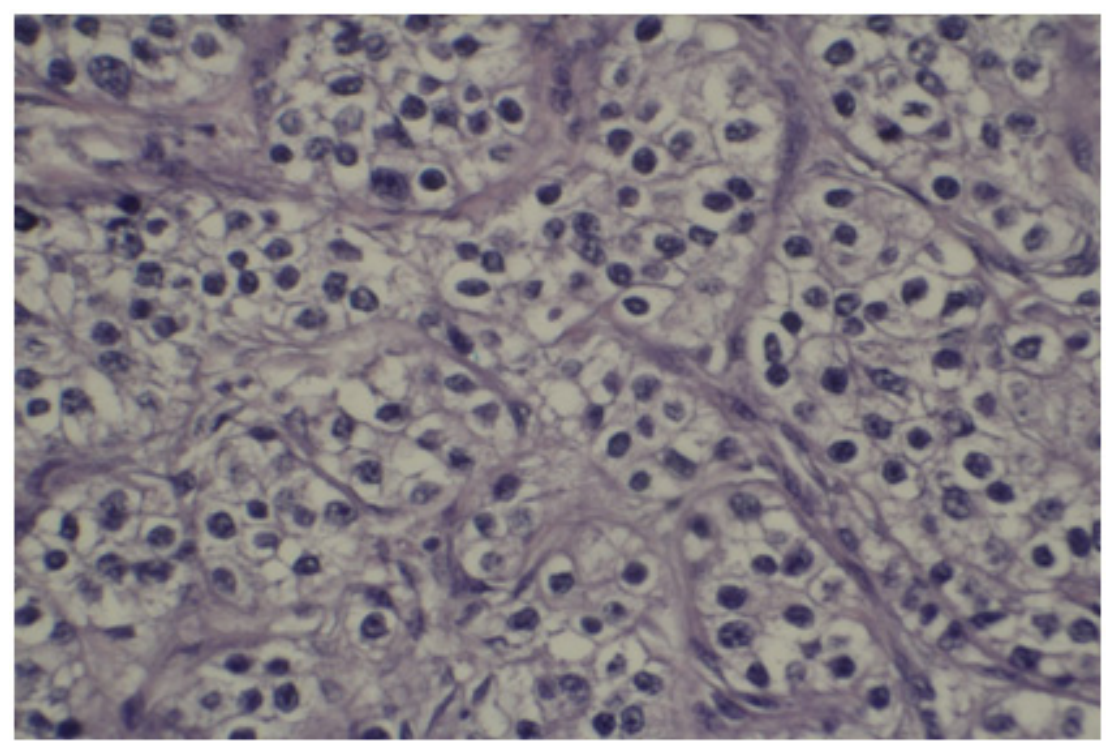

Figura 4: Lesão de arco costal com presença de hipercromasia com núcleos pleomórficos. 


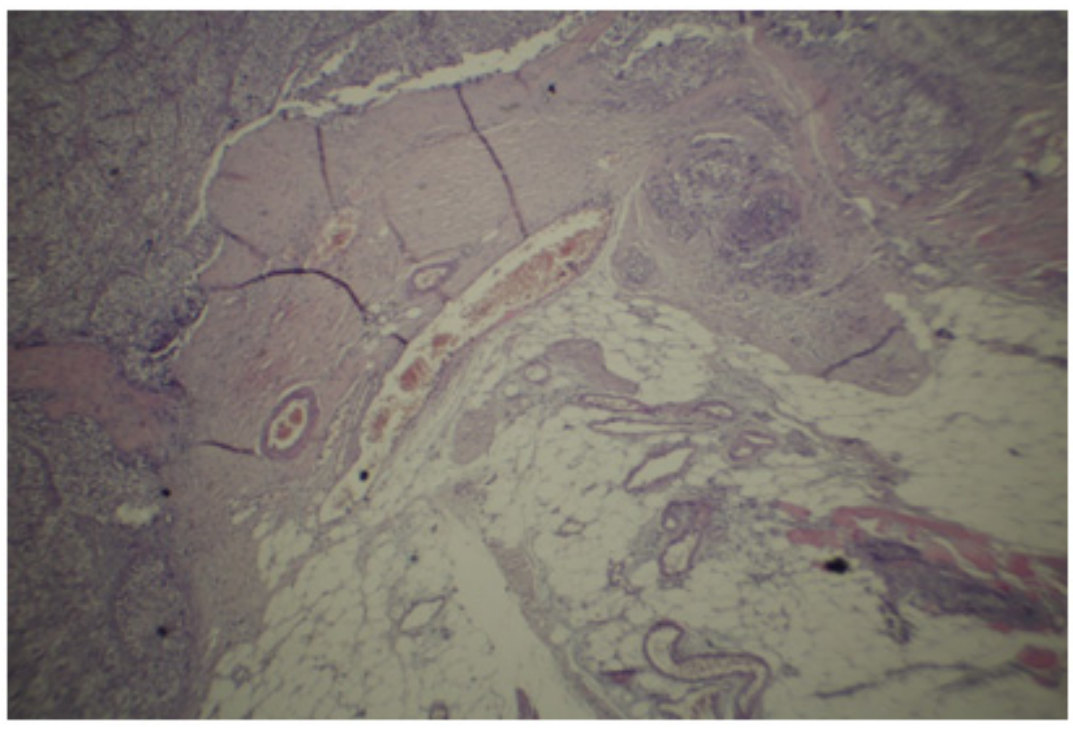

Figura 5: Presença de invasão fibroadiposa na lesão de arco costal.

Foi transferida para os cuidados da nefrologia em 17/12/16, para manejo de diureticoterapia e tratamento de acidose metabólica hiperclorêmica, possivelmente secundária a infusão excessiva de Soro Fisiológico $0,9 \%$ ou acidose tubular renal tipo IV. A terapia oral com furosemida foi mantida e otimizada a reposição de bicarbonato de cálcio. A função renal encontrava-se estável, com discreta tendência a melhora. Realizado USG abdominal a beira do leito, que evidenciou rins eutópicos, volume reduzido com hiperecogenicidade cortical e aumento da diferenciação cortico-medular com imagem sugerindo calcificações em pirâmides renais à direita.

A paciente recebeu alta hospitalar em 22/12/ 16 em regular estado geral, com diurese preservada, subnutrida, porém com boa aceitação a dieta oral. Encontra-se em acompanhamento ambulatorial no Centro de Especialidades Médicas (CEM) pela endocrinologia e pela nefrologia do Hospital Santa Casa de Belo Horizonte.

\section{Comentários}

Por constituir doença incomum e detentora de peculiaridades propedêutico-terapêuticas, O CP suscita importante interesse médico. Em geral, apresenta-se como hiperparatireoidismo primário sintomático, de curso evidente e dramático no que tange em evolução e gravidade. Esta neoplasia, cuja incidência anual é de 1,25 casos para cada $10,000,000$ de pessoas, corresponde a menos de $1 \%$ dos hiperparatireoidismos. ${ }^{8}$ De acordo com alguns relatos, aparentemente é mais comum no Japão, cujos dados apontam $5 \%$ de incidência. ${ }^{9} \mathrm{~A}$ etiologia ainda é desconhecida, sendo alguns casos relatados em pacientes com histórico de irradiação cervical e tende a recorrer localmente e disseminar-se para estruturas contíguas cervicais.

A associação entre gênero e carcinoma paratireoide não ocorre, embora no hiperparatireoidismo primário haja notável predominância feminina (razão de 3-4:1). A maioria dos pesquisadores observou que a idade média do paciente com CP está na quinta década, aproximadamente 10 anos antes dos pacientes com hiperparatireoidismo primário. ${ }^{1}$ No presente caso, a paciente apresentou quadro na quarta década de vida, demonstrando uma precocidade de hiperparatireoidimo primário, sendo importante a investigação para etiologia neoplásica.

As alterações genéticas que levam ao desenvolvimento do CP ainda não são bem compreendidas. Embora a hiperplasia da paratireoide esteja bem documentada em síndromes hereditárias, como Neoplasia Endócrina tipo 1 e 2A, no caso de CP isso é raramente observado. ${ }^{10}$ Contudo, de 10 a $15 \%$ dos pacientes com tumores hereditários de hiperparatireoidismo (HPT-JT), associada a mutações no 
gen supressor tumoral HRPT2, são diagnosticados com $\mathrm{CP}^{11}$. Isso sugere que o HRPT2 pode ser importante no desenvolvimento do CP.12

As apresentações clínicas do CP são variadas, sendo evidente a presença de quadros clínicos muito mais sintomáticos. No presente caso, a sintomatologia mais exuberante eram sintomas constitucionais e perda ponderal expressiva, que é corroborado pela literatura. Em um dos estudos retrospectivos de 43 casos de carcinoma, Wynne et al (1992) observaram como apresentação inicial da doença $38 \%$ de poliúria e polidipsia, $27 \%$ de mialgias ou artralgias, $17 \%$ de perda ponderal, $10 \%$ de nefrolitíase e 7\% assintomáticos. A prevalência de nefrolitíase foi de $56 \%$ e de insuficiência renal foi de $84 \% .{ }^{13}$ Outros estudos mostraram prevalência de envolvimento renal, incluindo nefrolitíase, nefrocalcinose e lesões de infiltração glomerular, inferior a $20 \% .{ }^{14} \mathrm{~A}$ paciente do caso em questão apresentou declínio da função renal, que pode ser associada às calcificações renais presentes, apesar de não haver relato de urolitíases e quadros relacionados a tal. No presente caso, não havia massa cervical palpável, porém sua presença foi reportada em estudos com variação de 30 a $76 \%$ de apresentação nos casos.

As metástases ocorrem tardiamente, com propagação por via linfática e hematogênica. Nódulos cervicais $(30 \%)$ e pulmonares $(40 \%)$ ocorrem mais comumente, seguido por lesões hepáticas $(10 \%)$. A paciente desse relato apresentou lesões osteolíticas em diversos segmentos ósseos compatível com tumor marrom, porém com uma lesão em arco costal de padrão heterogêneo, a qual foi investigada.

Os sinais radiológicos do hiperparatireoidismo, tais como osteíte fibrosa, reabsorção óssea subperiosteal, crânio em "sal e pimenta" e ausência de lâmina dura, bem como sinais menos específicos, como osteopenia espinhal difusa, são comumente observados no CP (44-91\%). ${ }^{1}$

Do ponto de vista laboratorial, o perfil corresponde à clínica: níveis muito elevados de calcemia, em geral superiores a $14,0 \mathrm{mg} / \mathrm{dL}$ ou $3-4$ vezes o limite superior da normalidade, e de paratormônio, muitas vezes superiores a $500 \mathrm{ng} / \mathrm{L}$ ou 3-10 vezes o limite superior da normalidade. ${ }^{15}$ Os outros parâmetros laboratoriais também acompanham este perfil, com hipofosfatemia frequente, elevação dos marcadores bioquímicos do metabolismo ósseo e hipercalciúria importante. Alterações radiológicas ósseas são comuns e típicas da osteíte fibrosa. Os pacientes com CP podem ter níveis elevados de alpha e beta- subunidades de hCG, enquanto que nos pacientes com hiperparatireoidismo primário não se observa. ${ }^{16}$

No presente caso, a paciente apresentava manutenção de níveis de hipercalcemia PTH-dependente, com atenção especial para a dosagem de PTH da lesão de arco costal, cujo nível era muitíssimo elevado. A dosagem desse marcador em lesão possivelmente metastática é inovador, não sendo encontrados relatos na literatura médica.

Yip et al (2008) relatam que a maioria dos pacientes apresenta diminuição, apropriada, dos níveis de PTH dentro de 10 minutos após a remoção do tumor ${ }^{17}$. Destacam ainda que o percentual de pacientes com achados operatórios suspeitos de CP (massa dura, aderência ou invasão em estruturas adjacentes, ou fibrose) foram efetivamente avaliados como CP por critérios histológicos rigorosos: presença de invasão angiolinfática, perineural, extracapsular ou tireoidiana, metástase ou mitoses atípicas. A suspeita clínica de CP é, portanto, importante na determinação da extensão da ressecção, embora não seja definitiva em termos diagnósticos.

Do ponto de vista anatomopatológico, a definição de carcinoma de paratireoide nem sempre é simples. Baseia-se, em seu caráter macroscópico, no encontro de tumores grandes, com diâmetros superiores a $1,5 \mathrm{~cm}$, aderentes aos tecidos adjacentes e frequentemente apresentando invasão do lobo tireoidiano ipsilateral e musculatura cervical. ${ }^{18}$ Na microscopia, os carcinomas apresentam bandas fibrosas acelulares espessas, atividade mitótica, invasão vascular e invasão capsular. ${ }^{19}$ Muitas vezes a diferenciação não é muito característica, e alguns aspectos podem ser duvidosos, sugerindo imagens "suspeitas" de carcinoma.

O carcinoma é composto por células pleomórficas, geralmente de volume maior do que as do adenoma, sendo que frequentemente as células se encontram agrupadas em torno de capilares de paredes finas, ${ }^{13}$ sendo que fibrose, necrose, atipia nuclear e figuras de mitose são mais frequentes no carcinoma, mas não são patognomônicas e nem achados consistentes de processo maligno. ${ }^{20} \mathrm{~A}$ distinção patológica entre o carcinoma e o adenoma 
de paratireoide é difícil, uma vez que pleomorfismo nuclear, hipercromatismo, células tumorais livres nos vasos sanguíneos, núcleos bizarros e células gigantes podem ser encontradas em $25 \%$ dos adenomas e nem todos os carcinomas apresentam figuras de mitoses. A invasão capsular e vascular parece ser o único fato que na patologia distingue inequivocamente o carcinoma do adenoma. Tais achados corroboram a dificuldade para determinação da etiologia, como foi ilustrado no presente caso, em que ocorreu somente após a confirmação de lesão metastática em arco costal. Eventualmente, apenas a evolução, como não-cura, recidiva ou mesmo ocorrência de metástases, pode definir a malignidade, e uma revisão de material é necessária para nova classificação ${ }^{15}$. No presente caso, os achados da biopsia de paratireoides foi inconclusiva, em que havia sinais de processo neoplásico, não sendo possível estabelecer a etiologia maligna de tal processo.

Segundo os relatos de literatura, a sensibilidade da cintilografia com tálio e tecnécio é de $73 \%$, da TC de $68 \%$, da USG cervical de $55 \%$ e da Ressonância Magnética de $57 \%$, sendo que a especificidade relatada é de $94 \%, 92 \%, 95 \%$ e $87 \%$, respectivamente, e a sensibilidade aumenta quando existe a combinação entre cintilografia e tomografia. ${ }^{21}$ Mais recentemente, estudos clínicos com [18F]FDG (fluorodesóxiglicose) têm demonstrado resultados promissores no carcinoma de paratireoide. ${ }^{22} \mathrm{~A}$ cintilografia com Tc- 99m MDP (tecnécio) é bastante sensível, mas não específica, sendo a captação do esqueleto proporcional ao fluxo regional, formação de osteoide e mineralização. O Tc 99m MIBI (metil-iodobenzilguanidina) pode ser usado para detectar lesões de paratireoide, incluindo carcinoma e tumor marrom em pacientes com dores ósseas e achados que mimetizam doença metastática, bem como no seguimento de tumores marrons após a terapia cirúrgica para $\mathrm{CP}^{23}$ De forma não usual, a paciente em questão não apresentou alterações às cintilografias realizadas, o que demonstra que apesar da sensibilidade desse exame ser significativa, alguns casos podem ser falso-negativos. Desse modo, dados clínicos, complementares e anatomopatológicos são essências para se firmar o diagnóstico dessa neoplasia de incidência tão baixa.

A suspeita pré-operatória e intraoperatória é importante no planejamento do tratamento cirúrgico eficaz: a ressecção completa do tumor na pri- meira exploração é relatada com melhores chances de cura. O melhor tratamento cirúrgico é a ressecção "en bloc" com lobectomia tireoideana ipsilateral e esvaziamento linfonodal, em casos de aumento ou anormalidades em gânglios. A maioria dos autores enfatiza a importância de uma abordagem inicial agressiva para redução de recorrências locais e a distância. ${ }^{1,5}$ Deve-se dispensar particular atenção para evitar a ruptura do tumor durante 0 ato cirúrgico, devido ao risco muito alto de recorrência local e doença persistente ou recorrente. ${ }^{1,24}$

No presente caso, não se adotou uma conduta cirúrgica agressiva, com exérese somente das paratireoides. Isso se deveu a inespecificidade de dados, clínico e radiológico, que corroborassem a existência de processo maligno, num primeiro momento. A exérese da lesão em arco costal, de padrão distinto, foi fundamental para se firmar a etiologia da doença, dado a altíssima concentração de PTH na lesão costal. A ocorrência de fome óssea após a exérese da lesão constitui mais um dado para o diagnóstico de CP. Salienta-se que o nível de PTH decresceu em $85 \%$ em 48 horas após a retirada da lesão metastática.

Recentemente, alguns autores relataram um papel para a radioterapia adjuvante. A irradiação adjuvante do pescoço e do mediastino tem sido relatada como sendo útil na redução do risco de progressão da doença loco-regional e na melhoria da sobrevida. ${ }^{25,26}$ Outros relatos sugerem um efeito positivo da radioterapia para sintomas paliativos de hipercalcemia em pacientes inoperáveis. ${ }^{27}$

O prognóstico do CP é bastante variável. Nenhuma característica se correlaciona previsivelmente com o desfecho. Diagnóstico e ressecção completa no ato cirúrgico inicial levam a melhor prognóstico. O tempo médio entre a cirurgia e a primeira recorrência é de aproximadamente três anos, apesar de terem sido relatados intervalos de até 20 anos. Uma vez recidivado, cura completa do tumor é improvável, embora a sobrevivência ainda seja comum com cirurgia paliativa. As taxas de sobrevivência em 05 anos variam de $40-86 \%$. A pesquisa do National Cancer Data base demonstrou sobrevivência de $49 \%$, em 10 anos. ${ }^{28}$

Uma análise de sobrevida para CP das bases de dados oncológicos, tais como "Surveillance, Epidemiology and End Results (SEER)", "National Cancer Data Base (NCDB)", e Swedish Cancer 
Registry e estudos longitudinais retrospectivos mostraram taxas de sobrevivência de $85 \%$ e $49-77 \%$ em seguimento de 5 e 10 anos, respectivamente. ${ }^{29}$

\section{Conclusão}

O carcinoma de paratireoide constitui neoplasia incomum, dada a sua baixa incidência, sendo necessária alta suspeição clínica e laboratorial. O presente trabalho relata um caso de hiperpatireoidismo primário, sem massa cervical palpável e presença de lesão metastática em arco costal de CP, diagnosticado por dosagem de PTH de tal lesão. Os estudos com radionuclídeos não evidenciaram alterações em paratireoides e lesões ósseas, a despeito de metástases sem invasão local neoplásica. O prognóstico é variável, porém a identificação e ressecção do tumor e das lesões metastáticas são essências para controle da doença e morbimortalidade.

\section{Referências}

1. Shane E. Clinical review 122: parathyroid carcinoma. J Clin Endocrinol Metab. 2001;86:485-93.

2. Shane E, Bilezikian J. Parathyroid Carcinoma: a review of 62 patients. Endocr Rev 1982; 3: 218-26.

3. Lee PK, Jarosek SL, Virnig BA, Evasovich M, Tuttle TM. Trends in the incidence and treatment of parathyroid cancer in the United States. Cancer. 2007; 109:1736-41.

4. Hundahl SA, Fleming ID, Fremgen AM, Menck HR. Two hundred eighty-six cases of parathyroid carcinoma treated in the U.S. between 1985-1995. Cancer. 1999;86: 53844.

5. Kebebew E. Parathyroid Carcinoma. Curr Treat Options Oncol. 2001; 2: 347-54.

6. Montoro JRM, Fava AS, Sartini AL, Mattiola LR, Brito AJP, Costa GP. Carcinoma de Paratireoide. Arq Int Otorrinolaringol. 2008; 12:145-9.

7. Digonnet A, Carlier A, Willemse E, Quiriny M, Dekeyser C, Aubain NS, et al. Parathyroid carcinoma: a review with three illustrative cases. J Cancer. 2011;2:532-7, http:// dx.doi.org/10.7150/jca.2.532

8. Schaapveld M, Jorna FH, Aben KK, Haak HR, Plukker JT, Links TP. Incidence and prognosis of parathyroid gland carcinoma: a populationbased study in The Netherlands estimating the preoperative diagnosis. Am J Surg. 2011;202:590-7, http://dx.doi.org/10.1016/j.amjsurg. 2010.09.025.

9. Obara T, Fujimoto Y. Diagnosis and treatment of patients with parathyroid carcinoma: an update and review. World J Surg 1991;15:738-44.

10. Dionisi S, Minisola S, Pepe J, De Geronimo S, Paglia F, Memeo $\mathrm{L}$, et al. Concurrent parathyroid adenomas and carcinoma in the setting of multiple endocrine neoplasia type 1: presentation as hypercalcemic crisis. Mayo Clin Proc. 2002; 77:866-9.

11. Chen JD, Morrison C, Zhang C, Kahnoski K, Carpten JD, Teh BT. Hyperparathyroidism-jaw tumour syndrome. J Intern Med 2003;253:634-42.
12. Carpten JD, Robbins CM, Villablanca A, Forsberg L, Presciuttini S, Bailey-Wilson J, et al. HRPT2, encoding parafibromin, is mutated in hyperparathyroidism-jaw tumor syndrome. Nat Genet. 2002;32:676-80.

13. Wynne AG, van Heerden J, Carney JA, Fitzpatrick LA. Parathyroid carcinoma: Clinical and Pathologic Features in 43 Pacients. Medicine (Baltimore). 1992;71:197-205.

14. Heath H, Hodgson S, Kennedy M. 1980 Primary hyperparathyroidism: incidence, morbidity, and potential economic impact in a community. N Engl J Med. 302:189-93.

15. Vieira JG, Ohe M, Hauache O, Oliveira U, Delana JM, Gonçalves A, Lazaretti-Castro M. Carcinoma de paratireoide. Arq Bras Endocrinol Metab. 2005; 49: 811-15.

16. Stock JL, Weintraub BD, Rosen SW, Aurbach GD, Spiegel AM, Marx SJ. Human chorionic gonadotropin subunit measurement in primary hyperparathyroidism. J Clin Endocrinol Metab. 1982; 54:57-63.

17. Yip L, Seetala R, Nikiforova M, Kikiforov $Y$, Ogilvie JB, Carty SE, et al. Loss of heterozygosity of selected tumor suppressor genes in parathyroid carcinoma. Surgery. 2008; $144 ; 949-55$

18. Wang C, Gaz R. Natural history of parathyroid carcinoma: diagnosis, treatment and results. Am J Surg. 1985; 149:522-7.

19. Schantz A, Caltelman B. Parathyroid carcinoma: a study of 70 cases. Cancer. 1973;31:600-5.

20. Scully RE, Mark EJ, McNeely WF, Ebeling SH. Parathyroid carcinoma: case records of the Massachussets general hospital. N Engl J Med. 1996; 335:1213-20.

21. Krubsack AJ, Wilson SD, Lawson TL, Kneeland JB, Thorsen MK, CollierD, et al. Prospective comparison of radionuclide, computed tomographic, sonographic and magnetic resonance localization of parathyroid tumors. Surgery. 1989;106:639-46.

22. Neumann DR, Esselstyn CB, Kim EY. Recurrent postoperative parathyroid carcinoma; FDG-PET and SestamibiSPECT findings. J Nucl Med.1996;37:2000-1.

23. Pai M, Park $\mathrm{CH}$, Kim BS, Chung YS, Park HB. Multiple brown tumors in parathyroid carcinoma mimicking metastatic bone disease. Clin Nucl Med. 1997;22:691-4.

24. Sandelin K, Thompson NW, Bondeson L. Metastatic parathyroid carcinoma dilemmas in management. Surgery. 1991;110:978-88.

25. Clayman GL, Gonzales HE, El Naggar A, Vassilopoulou R. Parathyroid carcinoma: evaluation and interdisciplinary management. Cancer. 2004;100:900-5. doi: 10.1002/ cncr.20089.

26. Munson ND, Foot RL, Northcutt RC, Tiegs RD, Fitzpatrick LA, Grant CS, van Heerden JA, Thompson GB, Lloyd RV. Parathyroid carcinoma: is there a role for adjuvant radiation therapy? Cancer. 2003;98:2378-84. doi: 10.1002/ cncr.11819.

27. Rasmuson T, Kristoffersson A, Boquist L. Positive effect of radiotherapy ad surgery on hormonally active pulmonary metastases of primary parathyroid carcinoma. Eur J Endocrinol. 2000;143:749-754. doi: 10.1530/ eje.0.1430749

28. Hundahl SA, Fleming ID, Fremgen AM, Menck HR. 1999 Two hundred eighty-six cases of parathyroid carcinoma treated in the U.S. between 1985- 1995: a National Cancer Data Base Report. The American College of Surgeons Commission on Cancer and the American Cancer Society. Cancer. 1999; 86:538-44.

29. Wei $\mathrm{CH}$, Harari A. Parathyroid carcinoma: update and guidelines for management. Curr Treat Options Oncol. 2012;13:11-23, http://dx.doi. org/10.1007/s11864-0110171-3. 